\title{
FINITE-DIMENSIONAL DISTRIBUTIONS OF A SQUARE-ROOT DIFFUSION
}

\author{
MICHAEL B. GORDY, ${ }^{*}$ Federal Reserve Board
}

\begin{abstract}
We derive multivariate moment generating functions for the conditional and stationary distributions of a discrete sample path of $n$ observations of a square-root diffusion (CIR) process, $X(t)$. For any fixed vector of observation times $t_{1}, \ldots, t_{n}$, we find the conditional joint distribution of $\left(X\left(t_{1}\right), \ldots, X\left(t_{n}\right)\right)$ is a multivariate noncentral chi-squared distribution and the stationary joint distribution is a KrishnamoorthyParthasarathy multivariate gamma distribution. Multivariate cumulants of the stationary distribution have a simple and computationally tractable expression. We also obtain the moment generating function for the increment $X(t+\delta)-X(t)$, and show that the increment is equivalent in distribution to a scaled difference of two independent draws from a gamma distribution.

Keywords: Bell polynomial; CIR process; difference of gamma variates; Kibble-Moran distribution; Krishnamoorthy-Parthasarathy distribution; multivariate noncentral chisquared distribution; multivariate gamma distribution; square-root diffusion
\end{abstract}

2010 Mathematics Subject Classification: Primary 60G17

Secondary 60E 10

\section{Introduction}

Let $X_{t}$ be a one-dimensional Feller (1951) square-root diffusion process with stochastic differential equation

$$
\mathrm{d} X_{t}=\left(\mu-\kappa X_{t}\right) \mathrm{d} t+\sigma \sqrt{X_{t}} \mathrm{~d} W_{t},
$$

where $W_{t}$ is a Brownian motion. We assume that $\mu>0$ to ensure that $X_{t}$ remains nonnegative. This process is widely used in economics and finance, especially in modeling interest rates and corporate credit risk, where it is usually known as the CIR process (Cox et al. (1985)). In this paper we derive moment generating functions for the conditional and stationary multivariate distributions of a discrete sample path of this process.

Let $\boldsymbol{X} \equiv\left(X\left(t_{1}\right), \ldots, X\left(t_{n}\right)\right)$ be a discrete sample path for a given vector of ordered observation times $t_{1}<t_{2}<\ldots<t_{n}$. In Section 2 we derive the conditional joint moment generating function for $\boldsymbol{X}$ given $X\left(t_{0}\right)$ for $t_{0}<t_{1}$, and show that the conditional distribution is a multivariate noncentral chi-squared distribution of the type studied by Jensen (1969).

If we impose $\kappa>0$, then $X_{t}$ is stationary. In Section 3 we demonstrate that the stationary distribution of $\boldsymbol{X}$ is a Krishnamoorthy-Parthasarathy (1951) multivariate gamma distribution. (When $4 \mu / \sigma^{2}>n-1, \boldsymbol{X}$ can also be represented as the diagonal vector of a Wishart matrix (see Kotz et al. (2000, Section 48.3.3)). Series solutions for the density and cumulative distribution functions are given by Royen (1994) for a restricted class of the Krishnamoorthy-Parthasarathy distribution. We demonstrate that the distribution of $\boldsymbol{X}$ falls within this class. We also provide a simple and computationally tractable solution for the multivariate cumulants.

Received 18 April 2013; revision received 6 January 2014.

* Postal address: Federal Reserve Board, Washington, DC, 20551, USA. Email address: michael.gordy@ frb.gov 
In the $n=2$ case, the stationary distribution is a Kibble-Moran bivariate gamma distribution (see Kotz (2000, Section 48.2.3))). In Section 4 we study the stationary distribution of the increment $X(t+\delta)-X(t)$ for a fixed time-step $\delta$. We show that this increment is equivalent in distribution to a scaled difference between two independent gamma variates and provide a simple closed-form solution for the moments of this distribution. Other applications are discussed in Section 5.

\section{Conditional finite-dimensional distribution}

We derive the conditional moment generating function (MGF) $M_{\boldsymbol{X}}\left(\boldsymbol{u} \mid X\left(t_{0}\right)\right)$ for $\boldsymbol{X}$ given $X\left(t_{0}\right)$, where $\boldsymbol{u}$ denotes the vector of auxiliary variables $u_{1}, \ldots, u_{n}$. We assume $X\left(t_{0}\right) \geq 0$. It is well known that the transition distribution for $X(t+\delta)$ given $X(t)$ is a scaled noncentral chi-squared distribution (e.g. Alfonsi (2010)). Letting $M_{c}$ denote the conditional MGF for $X(t+\delta)$ given $X(t)$, we have

$$
M_{c}(u ; \delta, x)=\mathbb{E}[\exp (u X(t+\delta)) \mid X(t)]=(1-\theta u)^{-2 \mu / \sigma^{2}} \exp \left(\frac{\mathrm{e}^{-\kappa \delta} u}{1-\theta u} X(t)\right),
$$

where

$$
\theta= \begin{cases}\frac{\sigma^{2}}{2} \delta & \text { if } \kappa=0 \\ \frac{\sigma^{2}}{2 \kappa}(1-\exp (-\kappa \delta)) & \text { otherwise. }\end{cases}
$$

As the square-root diffusion is a Markov process, we have

$$
\begin{aligned}
\mathbb{E}\left[\exp \left(u_{k} X\left(t_{k}\right)\right) \mid X\left(t_{k-1}\right), X\left(t_{k-2}\right), \ldots, X\left(t_{1}\right), X\left(t_{0}\right)\right] & =\mathbb{E}\left[\exp \left(u_{k} X\left(t_{k}\right)\right) \mid X\left(t_{k-1}\right)\right] \\
& =M_{c}\left(u_{k} ; t_{k}-t_{k-1}, X\left(t_{k-1}\right)\right)
\end{aligned}
$$

for $k=1, \ldots, n$. For notational convenience, we define $\rho_{i, j}=\exp \left(-\kappa\left|t_{i}-t_{j}\right|\right)$ and

$$
\theta_{i, j}= \begin{cases}\frac{\sigma^{2}}{2}\left|t_{i}-t_{j}\right| & \text { if } \kappa=0, \\ \frac{\sigma^{2}}{2 \kappa}\left(1-\rho_{i, j}\right) & \text { otherwise }\end{cases}
$$

for $(i, j) \in\{0,1, \ldots, n\}^{2}$. We write $M_{\boldsymbol{X}}$ in nested form

$$
\begin{aligned}
M_{\boldsymbol{X}}\left(\boldsymbol{u} \mid X\left(t_{0}\right)\right) & \\
= & \mathbb{E}\left[\exp (\langle\boldsymbol{u}, \boldsymbol{X}\rangle) \mid X\left(t_{0}\right)\right] \\
= & \mathbb{E}\left[\exp \left(\sum_{k=1}^{n-1} u_{k} X\left(t_{k}\right)\right) M_{c}\left(u_{n} ; t_{n}-t_{n-1}, X\left(t_{n-1}\right)\right) \mid X\left(t_{0}\right)\right] \\
= & \left(1-\theta_{n-1, n} u_{n}\right)^{-2 \mu / \sigma^{2}} \mathbb{E}\left[\exp \left(\sum_{k=1}^{n-2} u_{k} X\left(t_{k}\right)\right)\right. \\
& \left.\times \mathbb{E}\left[\exp \left(u_{n-1} X\left(t_{n-1}\right)\right) \exp \left(\frac{\rho_{n-1, n} u_{n}}{1-\theta_{n-1, n} u_{n}} X\left(t_{n-1}\right)\right) \mid X\left(t_{n-2}\right)\right] \mid X\left(t_{0}\right)\right] \\
= & \left(1-\theta_{n-1, n} u_{n}\right)^{-2 \mu / \sigma^{2}} \mathbb{E}\left[\exp \left(\sum_{k=1}^{n-2} u_{k} X\left(t_{k}\right)\right) M_{c}\left(\tilde{u}_{n-1} ; t_{n-1}-t_{n-2}, X\left(t_{n-2}\right)\right) \mid X\left(t_{0}\right)\right],
\end{aligned}
$$


where

$$
\tilde{u}_{n-1}=u_{n-1}+\frac{\rho_{n-1, n} u_{n}}{1-\theta_{n-1, n} u_{n}} .
$$

Repeating this process $n$ times in total, we obtain

$$
M_{\boldsymbol{X}}\left(\boldsymbol{u} \mid X\left(t_{0}\right)\right)=\left[\prod_{k=1}^{n}\left(1-\theta_{k-1, k} \tilde{u}_{k}\right)\right]^{-2 \mu / \sigma^{2}} \exp \left(\tilde{u}_{0} X\left(t_{0}\right)\right),
$$

where the modified auxiliary variables have the forward recursive relationship

$$
\tilde{u}_{k}=u_{k}+\frac{\rho_{k, k+1} \tilde{u}_{k+1}}{1-\theta_{k, k+1} \tilde{u}_{k+1}}
$$

for $k=0, \ldots, n$ and where we fix $u_{0}=0$ and $\tilde{u}_{n+1}=0$.

We will express (2.1) in a compact matrix form. We first establish the notation and preliminary results. A vector $\boldsymbol{a}$ of length $m$ is positive-decreasing if $a_{1}>a_{2}>\cdots>a_{m}>0$.

Definition 2.1. (Correlation matrix generated by $\boldsymbol{a}$.) Given a positive-decreasing vector $\boldsymbol{a}$ of length $m$, let $C(\boldsymbol{a})$ be the $m \times m$ matrix with elements

$$
C(\boldsymbol{a})[i, j]=\frac{a_{\max \{i, j\}}}{a_{\min \{i, j\}}} .
$$

The matrix $C(\boldsymbol{a})$ is a one-pair matrix in the sense of Gantmacher and Kreĭn (see Vandebril et al. (2010, Definition 3.11)), which leads to the following properties.

Lemma 2.1. If vector a of length $m$ is positive-decreasing then

(i) the determinant of $C(\boldsymbol{a})$ is

$$
\operatorname{det}(C(\boldsymbol{a}))=\prod_{k=1}^{m-1}\left(1-C(\boldsymbol{a})[k, k+1]^{2}\right)=\prod_{k=1}^{m-1}\left(1-\frac{a_{k+1}^{2}}{a_{k}^{2}}\right)>0 ;
$$

(ii) the inverse of $C(\boldsymbol{a})$ is a symmetric tridiagonal matrix with nonzero elements

$$
\begin{aligned}
C(\boldsymbol{a})^{-1}[k, k] & =\frac{a_{k}^{2}\left(a_{k-1}^{2}-a_{k+1}^{2}\right)}{\left(a_{k-1}^{2}-a_{k}^{2}\right)\left(a_{k}^{2}-a_{k+1}^{2}\right)} \\
C(\boldsymbol{a})^{-1}[k, k+1] & =C(\boldsymbol{a})^{-1}[k+1, k]=\frac{-a_{k} a_{k+1}}{a_{k}^{2}-a_{k+1}^{2}} ;
\end{aligned}
$$

where, for notational convenience, we define $a_{0}=\infty$ and $a_{m+1}=0$, and

(iii) the product $\boldsymbol{a} C(\boldsymbol{a})^{-1}$ is a vector with first element $a_{1}$ and zero remaining elements.

Proof. The expression for the determinant (i) follows directly from Proposition 3.16 of Vandebril et al. (2010). The inverse (ii) follows directly from Roy et al. (1960, Section 3), see also Vandebril et al. (2010, Theorem 3.17), and (iii) is straightforward to verify from (ii).

We call $C(\boldsymbol{a})$ a correlation matrix because it is symmetric positive definite for all on- and off-diagonal elements bounded in $(0,1)$.

Let $\beta_{i, j}=\sqrt{\rho_{i, j} / \theta_{i, j}}$, and for $m=1, \ldots, n$ let $\boldsymbol{b}_{m}$ be the length- $m$ row vector

$$
\boldsymbol{b}_{m}=\left[\beta_{n-m, n-m+1}, \beta_{n-m, n-m+2}, \ldots, \beta_{n-m, n}\right] .
$$

For each $m$, the elements of $\boldsymbol{b}_{m}$ are positive-decreasing, so $R_{m} \equiv C\left(\boldsymbol{b}_{m}\right)$ is a correlation matrix. 
Let $\boldsymbol{I}_{m}$ be the identity matrix, and $\Upsilon_{m}$ the diagonal matrix

$$
\Upsilon_{m} \equiv \operatorname{diag}\left(\left[\theta_{n-m, n-m+1} u_{n-m+1}, \theta_{n-m, n-m+2} u_{n-m+2}, \ldots, \theta_{n-m, n} u_{n}\right]\right) .
$$

The main result of this section is given in Theorem 2.1.

Theorem 2.1. The conditional MGF of $\left(X\left(t_{1}\right), \ldots, X\left(t_{n}\right)\right)$ given $X\left(t_{0}\right)$ is

$$
M_{\boldsymbol{X}}\left(\boldsymbol{u} \mid X\left(t_{0}\right)\right)=\operatorname{det}\left(\boldsymbol{I}_{n}-R_{n} \Upsilon_{n}\right)^{-2 \mu / \sigma^{2}} \exp \left(\boldsymbol{b}_{n} \Upsilon_{n}\left(\boldsymbol{I}_{n}-R_{n} \Upsilon_{n}\right)^{-1} \boldsymbol{b}_{n}^{\prime} X\left(t_{0}\right)\right) .
$$

Proof. We demonstrate that the expression on the right-hand side is equivalent to (2.1). We first show by induction that

$$
\tilde{u}_{n-m}-u_{n-m}=\boldsymbol{b}_{m} \Upsilon_{m}\left(\boldsymbol{I}_{m}-R_{m} \Upsilon_{m}\right)^{-1} \boldsymbol{b}_{m}^{\prime} \equiv q_{m}
$$

for $m=1, \ldots, n$. For $m=1$,

$$
q_{1}=\boldsymbol{b}_{1} \Upsilon_{1}\left(\boldsymbol{I}_{1}-R_{1} \Upsilon_{1}\right)^{-1} \boldsymbol{b}_{1}^{\prime}=\frac{\beta_{n-1, n}^{2} \theta_{n-1, n} u_{n}}{1-\theta_{n-1, n} u_{n}}=\frac{\rho_{n-1, n} u_{n}}{1-\theta_{n-1, n} u_{n}}=\tilde{u}_{n-1}-u_{n-1},
$$

where the last equality follows from $u_{n}=\tilde{u}_{n}$ and (2.2).

Now assume that (2.3) holds for all $1 \leq k<m$. Define $A_{m} \equiv R_{m}^{-1}-\Upsilon_{m}$ so that $\left(\boldsymbol{I}_{m}-\right.$ $\left.R_{m} \Upsilon_{m}\right)^{-1}=A_{m}^{-1} R_{m}^{-1}$. By Lemma 2.1(ii), $R_{m}^{-1}$ is symmetric tridiagonal, so $A_{m}$ is also symmetric tridiagonal. Let $\boldsymbol{\phi}_{m}$ be the vector

$$
\boldsymbol{\phi}_{m} \equiv \sqrt{\rho_{n-m, n-m+1}}\left[\frac{1}{\beta_{n-m, n-m+1}}, \frac{\beta_{n-m+1, n-m+2}}{\beta_{n-m, n-m+2}}, \frac{\beta_{n-m+1, n-m+3}}{\beta_{n-m, n-m+3}}, \ldots, \frac{\beta_{n-m+1, n}}{\beta_{n-m, n}}\right]
$$

and $\Phi_{m} \equiv \operatorname{diag}\left(\boldsymbol{\phi}_{m}\right)$. Define

$$
\begin{aligned}
& \hat{\boldsymbol{b}}_{m} \equiv \boldsymbol{b}_{m} \Phi_{m}, \quad \hat{\Upsilon}_{m} \equiv \Phi_{m}^{-1} \Upsilon_{m} \Phi_{m}^{-1}, \quad \hat{R}_{m} \equiv \Phi_{m} R_{m} \Phi_{m}, \\
& \hat{A}_{m} \equiv \Phi_{m}^{-1} A_{m} \Phi_{m}^{-1}=\hat{R}_{m}^{-1}-\hat{\Upsilon}_{m} .
\end{aligned}
$$

We can rewrite the quadratic form $q_{m}$ as

$$
\begin{aligned}
q_{m} & =\boldsymbol{b}_{m} \Upsilon_{m} A_{m}^{-1} R_{m}^{-1} \boldsymbol{b}_{m}^{\prime} \\
& =\hat{\boldsymbol{b}}_{m} \Phi_{m}^{-1} \Phi_{m} \hat{\Upsilon}_{m} \Phi_{m}\left(\Phi_{m} \hat{A}_{m} \Phi_{m}\right)^{-1} \Phi_{m} \hat{R}_{m}^{-1} \Phi_{m} \Phi_{m}^{-1} \hat{\boldsymbol{b}}_{m}^{\prime} \\
& =\hat{\boldsymbol{b}}_{m} \hat{\Upsilon}_{m} \hat{A}_{m}^{-1} \hat{R}_{m}^{-1} \hat{\boldsymbol{b}}_{m}^{\prime} .
\end{aligned}
$$

It is straightforward to verify that the transformed variables embed lagged values of the original variables

$$
\begin{aligned}
& \hat{\boldsymbol{b}}_{m}= \sqrt{\rho_{n-m, n-m+1}}\left[1, \boldsymbol{b}_{m-1}\right], \\
& \hat{R}_{m}^{-1}= {\left[\begin{array}{cc}
\frac{R_{m}^{-1}[1,1]}{\boldsymbol{\phi}_{m}[1]^{2}} & {\left[\frac{R_{m}^{-1}[1,2]}{\boldsymbol{\phi}_{m}[1] \boldsymbol{\phi}_{m}[2]}, \mathbf{0}_{m-2}\right]} \\
{\left[\begin{array}{cc}
\frac{R_{m}^{-1}[1,2]}{\boldsymbol{\phi}_{m}[1] \boldsymbol{\phi}_{m}[2]}, \mathbf{0}_{m-2}
\end{array}\right],} & R_{m-1}^{-1}
\end{array}\right], } \\
& \hat{\Upsilon}_{m}=\left[\begin{array}{cc}
u_{n-m+1} & \mathbf{0}_{m-1} \\
\mathbf{0}_{m-1}^{\prime} & \Upsilon_{m-1}^{\prime}
\end{array}\right] .
\end{aligned}
$$

From the relationship

$$
\theta_{i, j+1}-\rho_{j, j+1} \theta_{i, j}=\theta_{j, j+1}
$$


we obtain

$$
\frac{\beta_{i, j}^{2} \beta_{i, j+1}^{2}}{\beta_{i, j}^{2}-\beta_{i, j+1}^{2}}=\left(\frac{1}{\beta_{i, j+1}^{2}}-\frac{1}{\beta_{i, j}^{2}}\right)^{-1}=\left(\frac{\rho_{i, j} \rho_{i, j+1}}{\rho_{i, j} \theta_{i, j+1}-\rho_{i, j+1} \theta_{i, j}}\right)=\rho_{i, j} \beta_{j, j+1}^{2} .
$$

This leads to

$$
\frac{R_{m}^{-1}[1,2]}{\boldsymbol{\phi}_{m}[1] \boldsymbol{\phi}_{m}[2]}=-\beta_{n-m+1, n-m+2}
$$

which, by Lemma 2.1(iii), implies

$$
\left[\frac{R_{m}^{-1}[1,2]}{\boldsymbol{\phi}_{m}[1] \boldsymbol{\phi}_{m}[2]}, \mathbf{0}_{m-2}\right]=-\boldsymbol{b}_{m-1} R_{m-1}^{-1} .
$$

Equation (2.6) similarly leads to

$$
\frac{R_{m}^{-1}[1,1]}{\boldsymbol{\phi}_{m}[1]^{2}}=\frac{\theta_{n-m, n-m+2}}{\theta_{n-m, n-m+1} \theta_{n-m+1, n-m+2}} .
$$

With these identities we can partition the matrix $\hat{A}_{m}$ as

$$
\hat{A}_{m}=\left[\begin{array}{cc}
\frac{\theta_{n-m, n-m+2}}{\theta_{n-m, n-m+1} \theta_{n-m+1, n-m+2}}-u_{n-m+1} & -\boldsymbol{b}_{m-1} R_{m-1}^{-1} \\
-R_{m-1}^{-1} \boldsymbol{b}_{m-1}^{\prime} & A_{m-1}
\end{array}\right] .
$$

From Lemma 2.1(iii) we have

$$
\hat{\boldsymbol{b}}_{m} \hat{R}_{m}^{-1}=\boldsymbol{b}_{m} R_{m}^{-1} \operatorname{diag}\left(\boldsymbol{\phi}_{m}\right)^{-1}=\left[\frac{\beta_{n-m, n-m+1}}{\boldsymbol{\phi}_{m}[1]}, 0,0, \ldots, 0\right],
$$

which implies that only the first column of $\hat{A}_{m}^{-1}$ appears in the product $\hat{A}_{m}^{-1} \hat{R}_{m}^{-1} \hat{\boldsymbol{b}}_{m}^{\prime}$. By the standard formula for the inverse of a partitioned matrix, we have

$$
\hat{A}_{m}^{-1}[\cdot, 1]=\frac{1}{\hat{A}_{m} / A_{m-1}}\left[1, \quad \boldsymbol{b}_{m-1} R_{m-1}^{-1} A_{m-1}^{-1}\right]^{\prime},
$$

where $\hat{A}_{m} / A_{m-1}$ is the Schur complement of $A_{m-1}$ in $\hat{A}_{m}$. Substituting into (2.4), we obtain

$$
\begin{aligned}
q_{m} & =\frac{\beta_{n-m, n-m+1}}{\boldsymbol{\phi}_{m}[1]} \frac{\sqrt{\rho_{n-m, n-m+1}}}{\hat{A}_{m} / A_{m-1}}\left(u_{n-m+1}+\boldsymbol{b}_{m-1} \Upsilon_{m-1} A_{m-1}^{-1} R_{m-1}^{-1} \boldsymbol{b}_{m-1}^{\prime}\right) \\
& =\frac{\rho_{n-m, n-m+1}\left(u_{n-m+1}+q_{m-1}\right)}{\theta_{n-m, n-m+1}\left(\hat{A}_{m} / A_{m-1}\right)} .
\end{aligned}
$$

The denominator is expanded as

$$
\theta_{n-m, n-m+1}\left(\hat{A}_{m} / A_{m-1}\right)=\theta_{n-m, n-m+1}\left(\hat{A}_{m}[1,1]-b_{m-1} R_{m-1}^{-1} A_{m-1}^{-1} R_{m-1}^{-1} b_{m-1}^{\prime}\right) .
$$

The quadratic form can be written as

$$
\begin{aligned}
b_{m-1} R_{m-1}^{-1} A_{m-1}^{-1} R_{m-1}^{-1} b_{m-1}^{\prime}= & b_{m-1} \Upsilon_{m-1} A_{m-1}^{-1} R_{m-1}^{-1} b_{m-1}^{\prime} \\
& +b_{m-1}\left(R_{m-1}^{-1}-\Upsilon_{m-1}\right) A_{m-1}^{-1} R_{m-1}^{-1} b_{m-1}^{\prime} \\
= & q_{m-1}+\beta_{n-m+1, n-m+2}^{2},
\end{aligned}
$$


so

$$
\begin{aligned}
\theta_{n-m, n-m+1}\left(\hat{A}_{m} / A_{m-1}\right)= & \frac{\theta_{n-m, n-m+2}}{\theta_{n-m+1, n-m+2}}-\theta_{n-m, n-m+1}\left(u_{n-m+1}+q_{m-1}\right) \\
& -\theta_{n-m, n-m+1} \beta_{n-m+1, n-m+2}^{2} \\
= & \frac{\theta_{n-m, n-m+2}-\rho_{n-m+1, n-m+2} \theta_{n-m, n-m+1}}{\theta_{n-m+1, n-m+2}} \\
& -\theta_{n-m, n-m+1}\left(u_{n-m+1}+q_{m-1}\right) \\
= & 1-\theta_{n-m, n-m+1}\left(u_{n-m+1}+q_{m-1}\right),
\end{aligned}
$$

where the final equality follows from (2.5). Substituting into (2.7), we arrive at

$$
q_{m}=\frac{\rho_{n-m, n-m+1}\left(u_{n-m+1}+q_{m-1}\right)}{1-\theta_{n-m, n-m+1}\left(u_{n-m+1}+q_{m-1}\right)}=\frac{\rho_{n-m, n-m+1} \tilde{u}_{n-m+1}}{1-\theta_{n-m, n-m+1} \tilde{u}_{n-m+1}} .
$$

This establishes (2.3), which immediately implies that $\tilde{u}_{0}$ in (2.1) is simply $q_{n}$.

By (2.8), we have

$$
\prod_{k=1}^{n}\left(1-\theta_{k-1, k} \tilde{u}_{k}\right)=\prod_{m=1}^{n} \theta_{n-m, n-m+1}\left(\hat{A}_{m} / A_{m-1}\right) .
$$

Because $\hat{A}_{m} / A_{m-1}$ is scalar, the Schur complement decomposition of the determinant gives

$$
\hat{A}_{m} / A_{m-1}=\frac{\operatorname{det}\left(\hat{A}_{m}\right)}{\operatorname{det}\left(A_{m-1}\right)}=\frac{1}{\operatorname{det}\left(\Phi_{m}\right)^{2}} \frac{\operatorname{det}\left(A_{m}\right)}{\operatorname{det}\left(A_{m-1}\right)} .
$$

We also have

$$
\operatorname{det}\left(\Phi_{m}\right)^{2}=\frac{\operatorname{det}\left(\hat{R}_{m}\right)}{\operatorname{det}\left(R_{m}\right)}=\frac{\operatorname{det}\left(R_{m}^{-1}\right)}{\operatorname{det}\left(\hat{R}_{m}^{-1}\right)}=\frac{\operatorname{det}\left(R_{m}^{-1}\right)}{\operatorname{det}\left(R_{m-1}^{-1}\right)} \frac{1}{\hat{R}_{m}^{-1} / R_{m-1}^{-1}} .
$$

The Schur complement in the last term is

$$
\begin{aligned}
\hat{R}_{m}^{-1} / R_{m-1}^{-1} & =\frac{\theta_{n-m, n-m+2}}{\theta_{n-m, n-m+1} \theta_{n-m+1, n-m+2}}-b_{m-1} R_{m-1}^{-1} R_{m-1} R_{m-1}^{-1} b_{m-1}^{\prime} \\
& =\frac{\theta_{n-m, n-m+2}}{\theta_{n-m, n-m+1} \theta_{n-m+1, n-m+2}}-\beta_{n-m+1, n-m+2}^{2} \\
& =\frac{\theta_{n-m, n-m+2}-\rho_{n-m+1, n-m+2} \theta_{n-m, n-m+1}}{\theta_{n-m, n-m+1} \theta_{n-m+1, n-m+2}} \\
& =\frac{1}{\theta_{n-m, n-m+1}},
\end{aligned}
$$

where the last equality follows from (2.5). Substitute into (2.10) to obtain

$$
\begin{aligned}
\hat{A}_{m} / A_{m-1} & =\frac{1}{\theta_{n-m, n-m+1}} \frac{\operatorname{det}\left(R_{m-1}^{-1}\right)}{\operatorname{det}\left(R_{m}^{-1}\right)} \frac{\operatorname{det}\left(A_{m}\right)}{\operatorname{det}\left(A_{m-1}\right)} \\
& =\frac{1}{\theta_{n-m, n-m+1}} \frac{\operatorname{det}\left(R_{m} A_{m}\right)}{\operatorname{det}\left(R_{m-1} A_{m-1}\right)} \\
& =\frac{1}{\theta_{n-m, n-m+1}} \frac{\operatorname{det}\left(\boldsymbol{I}_{m}-R_{m} \Upsilon_{m}\right)}{\operatorname{det}\left(\boldsymbol{I}_{m-1}-R_{m-1} \Upsilon_{m-1}\right)} .
\end{aligned}
$$


Substituting into (2.9), we obtain a telescoping product that simplifies to

$$
\prod_{k=1}^{n}\left(1-\theta_{k-1, k} \tilde{u}_{k}\right)=\prod_{m=1}^{n} \frac{\operatorname{det}\left(\boldsymbol{I}_{m}-R_{m} \Upsilon_{m}\right)}{\operatorname{det}\left(\boldsymbol{I}_{m-1}-R_{m-1} \Upsilon_{m-1}\right)}=\operatorname{det}\left(\boldsymbol{I}_{n}-R_{n} \Upsilon_{n}\right)
$$

Thus, the expression in Theorem 2.1 matches (2.1).

\section{Stationary finite-dimensional distribution}

When $\kappa>0$, the square-root diffusion is stationary. We assume stationarity here and for the remainder of the paper. To derive the stationary MGF from the conditional MGF, we fix $t_{1}, \ldots, t_{n}$ and let $t_{0} \rightarrow-\infty$. For $i, j \in\{1, \ldots, n\}$, we have

$$
\begin{array}{clrl}
\lim _{t_{0} \rightarrow-\infty} \rho_{0, j}=0, & \lim _{t_{0} \rightarrow-\infty} \theta_{0, j}=\frac{\sigma^{2}}{2 \kappa} \equiv \bar{\theta}, & & \lim _{t_{0} \rightarrow-\infty} \Upsilon_{n}=\bar{\theta} \operatorname{diag}(\boldsymbol{u}) \\
\lim _{t_{0} \rightarrow-\infty} R_{n}[i, j]=\sqrt{\rho_{i, j}} \equiv \bar{R}[i, j], & \lim _{t_{0} \rightarrow-\infty} \boldsymbol{b}_{n}=\mathbf{0}_{n} .
\end{array}
$$

Since

$$
\lim _{t_{0} \rightarrow-\infty} \boldsymbol{I}_{n}-R_{n} \Upsilon_{n}=\boldsymbol{I}_{n}-\bar{\theta} \bar{R} \operatorname{diag}(\boldsymbol{u})
$$

remains nonsingular in the limit, while $\boldsymbol{b}_{n}$ converges to 0 , we have

$$
\lim _{t_{0} \rightarrow-\infty} \boldsymbol{b}_{n} \Upsilon_{n}\left(\boldsymbol{I}_{n}-R_{n} \Upsilon_{n}\right)^{-1} \boldsymbol{b}_{n}^{\prime}=0 .
$$

Thus, we have established the stationary MGF.

Theorem 3.1. The stationary $M G F$ of $\left(X\left(t_{1}\right), \ldots, X\left(t_{n}\right)\right)$ is

$$
M_{\boldsymbol{X}}(\boldsymbol{u})=\operatorname{det}\left(\boldsymbol{I}_{n}-\bar{\theta} \bar{R} \operatorname{diag}(\boldsymbol{u})\right)^{-2 \mu / \sigma^{2}} .
$$

In the preprint version of this paper (Gordy (2012)), Theorem 3.1 is proved by an alternative direct method and not as the limiting form of the conditional MGF.

The distribution of $\boldsymbol{X}$ is a special case of the broader class of Krishnamoorthy-Parthasarathy (1951) multivariate gamma distributions. The MGF in the general case is $\operatorname{det}(\boldsymbol{I}-C \operatorname{diag}(\boldsymbol{u}))^{-\alpha}$ for $\alpha>0$ and nonsingular $C$. It is usually (but not necessarily) assumed that each marginal distribution has unit scale, in which case the matrix $C$ has $1 \mathrm{~s}$ on the diagonal and $C[i, j]^{2}$ is the correlation between components $i$ and $j$. From this relationship, the matrix $C$ is known as the accompanying correlation matrix. In the specific case of the stationary square-root process, we could equivalently obtain the accompanying correlation matrix $\bar{R}$ from the known property of exponential decay in the autocorrelation function (Cont and Tankov (2004, Section 15.1.2)).

Series solutions for the density and cumulative distribution functions of the KrishnamoorthyParthasarathy distribution were derived by Royen (1994) for a class of correlation matrices (see also Kotz et al. (2000, Section 48.3.6)). This class includes any correlation matrix with tridiagonal inverse. Our matrix $\bar{R}$ is the correlation matrix generated by the vector

$$
\overline{\boldsymbol{b}} \equiv\left[\exp \left(-\left(\frac{\kappa}{2}\right) t_{1}\right), \exp \left(-\left(\frac{\kappa}{2}\right) t_{2}\right), \ldots, \exp \left(-\left(\frac{\kappa}{2}\right) t_{n}\right)\right]
$$

and, therefore, $\bar{R}^{-1}$ is tridiagonal by Lemma 2.1 . This property also allows for efficient computation of the determinant in the MGF. We can write

$$
\operatorname{det}\left(\boldsymbol{I}_{n}-\bar{\theta} \bar{R} \operatorname{diag}(\boldsymbol{u})\right)=\operatorname{det}\left(\bar{R}\left(\bar{R}^{-1}-\bar{\theta} \operatorname{diag}(\boldsymbol{u})\right)\right)=\operatorname{det}(\bar{R}) \cdot \operatorname{det}\left(\bar{R}^{-1}-\bar{\theta} \operatorname{diag}(\boldsymbol{u})\right) .
$$


Lemma 2.1 provides $\operatorname{det}(\bar{R})$. The matrix $\bar{R}^{-1}-\bar{\theta} \operatorname{diag}(\boldsymbol{u})$ is tridiagonal, which implies that the determinant satisfies a two-term recurrence rule (Horn and Johnson (1985, Section 0.9.10)). Consequently, the cost of computing the determinant is linear in $n$, whereas this cost is cubic in $n$ for a general matrix.

We next show that the multivariate cumulants of the distribution are easily calculated. Let $\boldsymbol{v}=\left(v_{1}, \ldots, v_{n}\right)$ be a vector of nonnegative integers with at least one positive element, and let $|\boldsymbol{v}|=\sum_{i=1}^{n} v_{i}$. Let $\Pi(k)$ be the set of permutations of $1, \ldots, k$ with the restriction $\pi(1)=1$. Our main result applies to any Krishnamoorthy-Parthasarathy distribution.

Theorem 3.2. Let $\boldsymbol{Y} \sim K P_{n}(\alpha, C)$. The cumulants of $\boldsymbol{Y}$ of order $\boldsymbol{v}$ are given by

$$
\psi_{\boldsymbol{v}}=\alpha \sum_{\pi \in \Pi(|\boldsymbol{v}|)} C[s(\pi(1)), s(\pi(|\boldsymbol{v}|))] \cdot C[s(\pi(|\boldsymbol{v}|)), s(\pi(|\boldsymbol{v}|-1))] \cdots C[s(\pi(2)), s(\pi(1))],
$$

where $\boldsymbol{s}$ is a vector of $v_{1}$ copies of 1 , followed by $v_{2}$ copies of 2, and so on.

Proof. The cumulant generating function is

$$
K_{\boldsymbol{Y}}(\boldsymbol{u})=\log \left(M_{\boldsymbol{Y}}(\boldsymbol{u})\right)=-\alpha \log (\operatorname{det}(\boldsymbol{I}-C \operatorname{diag}(\boldsymbol{u})))=-\alpha \log (\operatorname{det}(W(\boldsymbol{u}))),
$$

where we define $W(\boldsymbol{u})=\boldsymbol{I}-C \operatorname{diag}(\boldsymbol{u})$ for convenience.

Using $s$, we can write the cumulant of order $\boldsymbol{v}$ as $\psi_{v}=D_{u}^{s} K_{Y}(\mathbf{0})$, where

$$
D_{u}^{s} K_{\boldsymbol{Y}}(\boldsymbol{u})=\frac{\partial^{|\boldsymbol{v}|}}{\partial u_{s(1)} \cdots \partial u_{s(|\boldsymbol{v}|)}} K_{\boldsymbol{Y}}(\boldsymbol{u})
$$

We take partial derivatives in sequence, beginning with

$$
\frac{\partial}{\partial u_{s(1)}} K_{\boldsymbol{Y}}(\boldsymbol{u})=-\alpha \operatorname{tr}\left(W(\boldsymbol{u})^{-1} \frac{\partial W}{\partial u_{s(1)}}\right)=\alpha \operatorname{tr}\left(W(\boldsymbol{u})^{-1} C_{s(1)}\right),
$$

where we define $C_{k}$ as the $n \times n$ matrix matching $C$ on the $k$ th column and 0 elsewhere, i.e.

$$
C_{k}[i, j]= \begin{cases}C[i, k] & \text { if } j=k \\ 0 & \text { otherwise }\end{cases}
$$

Subsequent derivatives are

$$
\begin{gathered}
\frac{\partial^{2}}{\partial u_{s(1)} \partial u_{s(2)}} K_{\boldsymbol{Y}}(\boldsymbol{u})=\alpha \operatorname{tr}\left(W(\boldsymbol{u})^{-1} C_{s(2)} W(\boldsymbol{u})^{-1} C_{s(1)}\right), \\
\frac{\partial^{3}}{\partial u_{s(1)} \partial u_{s(2)} \partial u_{s(3)}} K_{\boldsymbol{Y}}(\boldsymbol{u})= \\
\alpha\left[\operatorname{tr}\left(W(\boldsymbol{u})^{-1} C_{s(3)} W(\boldsymbol{u})^{-1} C_{s(2)} W(\boldsymbol{u})^{-1} C_{s(1)}\right)\right. \\
\left.+\operatorname{tr}\left(W(\boldsymbol{u})^{-1} C_{s(2)} W(\boldsymbol{u})^{-1} C_{s(3)} W(\boldsymbol{u})^{-1} C_{s(1)}\right)\right] .
\end{gathered}
$$

Continuing this way, we arrive at

$$
\begin{array}{r}
D_{\boldsymbol{u}}^{s} K_{\boldsymbol{Y}}(\boldsymbol{u})=\alpha \sum_{\pi \in \Pi(|\boldsymbol{v}|)} \operatorname{tr}\left(W(\boldsymbol{u})^{-1} C_{S(\pi(|\boldsymbol{v}|))} W(\boldsymbol{u})^{-1} C_{S(\pi(|\boldsymbol{v}|-1))}\right. \\
\left.\cdots W(\boldsymbol{u})^{-1} C_{S(\pi(2))} W(\boldsymbol{u})^{-1} C_{S(\pi(1))}\right) .
\end{array}
$$


Observing that $W(\mathbf{0})=\boldsymbol{I}$, the cumulant can be written as

$$
\psi_{\boldsymbol{v}}=\alpha \sum_{\pi \in \Pi(|\boldsymbol{v}|)} \operatorname{tr}\left(C_{s(\pi(|\boldsymbol{v}|))} C_{s(\pi(|\boldsymbol{v}|)-1)} \cdots C_{S(\pi(2))} C_{S(\pi(1))}\right) .
$$

Because each $C_{k}$ matrix is nonzero only in column $k$, the product $C_{k} C_{\ell}$ of two such matrices has nonzero elements only in column $\ell$, and element $[i, \ell]$ is $C[i, k] \cdot C[k, \ell]$. This implies that the product $C_{S(\pi(|\boldsymbol{v}|))} C_{S(\pi(|\boldsymbol{v}|-1))} \cdots C_{S(\pi(2))} C_{S(\pi(1))}$ has nonzero elements only in the $s(\pi(1))$ column. The trace of the product is the $[s(\pi(1)), s(\pi(1))]$ element of the product and is given by

$$
\begin{aligned}
& \operatorname{tr}\left(C_{S(\pi(|\boldsymbol{v}|))} C_{S(\pi(|\boldsymbol{v}|-1))} \cdots C_{S(\pi(2))} C_{s(\pi(1))}\right) \\
& \quad=C[s(\pi(1)), s(\pi(|\boldsymbol{v}|))] \cdot C[s(\pi(|\boldsymbol{v}|)), s(\pi(|\boldsymbol{v}|-1))] \cdots C[s(\pi(2)), s(\pi(1))] .
\end{aligned}
$$

We substitute into (3.1) to complete the proof.

The set $\Pi(k)$ contains $(k-1)$ ! elements, so the cumulants are not too costly to compute for moderate values of $|\boldsymbol{v}|$. For vectors $\boldsymbol{v}$ containing large elements, computational efficiency can be improved by eliminating duplicated permutations of $s(\pi)$ in the summation. Say $m$ is the index of the smallest positive element of $\boldsymbol{v}$, i.e. $v_{m}$ satisfies

$$
v_{m}=\min \left(\max \left(v_{1}, 1\right), \ldots, \max \left(v_{n}, 1\right)\right) .
$$

Let $S_{\boldsymbol{v}, m}$ be the set of unique permutations of the vector $s$ with the restriction that $\tilde{s}(1)=m$ for all $\tilde{s} \in S_{\boldsymbol{v}, m}$. Then

$$
\psi_{\boldsymbol{v}}=\alpha \frac{\nu_{1} ! \nu_{2} ! \cdots v_{n} !}{v_{m}} \sum_{\tilde{s} \in S_{\boldsymbol{v}, m}} C[\tilde{s}(1), \tilde{s}(|\boldsymbol{v}|)] \cdot C[\tilde{s}(|\boldsymbol{v}|), \tilde{s}(|\boldsymbol{v}|-1)] \cdots C[\tilde{s}(2), \tilde{s}(1)] .
$$

For the cumulants of $\boldsymbol{X}$, we substitute $\alpha=2 \mu / \sigma^{2}$ and $\bar{\theta} \bar{R}$ for $C$. We exploit the exponential form of each element $\bar{R}[i, j]$ to obtain

$$
\psi_{\boldsymbol{v}}=\frac{2 \mu}{\sigma^{2}} \bar{\theta}^{|\boldsymbol{v}|} \frac{\nu_{1} ! \nu_{2} ! \cdots v_{n} !}{v_{m}} \sum_{\tilde{s} \in S_{\boldsymbol{v}, m}} \exp \left(-\left(\frac{\kappa}{2}\right) \sum_{i=1}^{|v|}\left|t_{\tilde{s}(i+1)}-t_{\tilde{s}(i)}\right|\right),
$$

where we define $\tilde{s}(|\boldsymbol{v}|+1)=\tilde{s}(1)=m$.

\section{Moments of the increments}

In the bivariate case, the stationary distribution simplifies to the Kibble-Moran bivariate gamma distribution with MGF as given in Corollary 4.1.

Corollary 4.1. The MGF of $(X(t), X(t+\delta))$ under stationarity is

$$
M_{X}\left(u_{1}, u_{2}\right)=\mathbb{E}\left[\exp \left(u_{1} X(t)+u_{2} X(t+\delta)\right)\right]=\left(\left(1-\bar{\theta} u_{1}\right)\left(1-\bar{\theta} u_{2}\right)-\bar{\theta}^{2} \rho u_{1} u_{2}\right)^{-2 \mu / \sigma^{2}},
$$

where $\rho=\exp (-\kappa \delta)$.

For fixed $\delta$, we call $X_{t+\delta}-X_{t}$ an increment of the process $X_{t}$. Under stationarity, $X_{t+\delta}-$ $X_{t} \stackrel{\text { D }}{=} X_{\delta}-X_{0}$ for all $t$, so without loss of generality we examine the stationary distribution of $\Delta_{\delta}=X_{\delta}-X_{0}$. 
From Corollary 4.1,

$$
\begin{aligned}
M_{\Delta}(u ; \delta) & =M_{\boldsymbol{X}}(-u, u) \\
& =\left(1-\bar{\theta}^{2}(1-\rho) u^{2}\right)^{-2 \mu / \sigma^{2}} \\
& =((1-\bar{\theta} \sqrt{1-\rho} u)(1+\bar{\theta} \sqrt{1-\rho} u))^{-2 \mu / \sigma^{2}} \\
& =M_{\Gamma}(u \sqrt{1-\rho}) \cdot M_{\Gamma}(-u \sqrt{1-\rho}),
\end{aligned}
$$

where $\rho=\exp (-\kappa \delta)$ and

$$
M_{\Gamma}(u)=(1-\bar{\theta} u)^{-2 \mu / \sigma^{2}}
$$

is the univariate stationary MGF for $X(t)$. An immediate implication of (4.1) is that $\Delta_{\delta}$ is equivalent in distribution to $(1-\rho)^{1 / 2}$ times $\Delta_{\infty}$. Furthermore, $\Delta_{\infty}$ is equivalent in distribution to the difference between two independent draws from the stationary distribution of $X(t)$. This gives a very simple method for sampling from the stationary distribution of $\Delta_{\delta}$.

Consider the general problem of the moments of the difference between two independent and identically distributed (i.i.d.) gamma variates. Let $Z_{1}, Z_{2} \stackrel{\text { i.i.d. }}{\sim} G a(\alpha, \beta)$ for shape parameter $\alpha>0$ and scale parameter $\beta>0$, and define $Y=Z_{1}-Z_{2}$. The $n$th cumulant of $Y$ is

$$
\psi_{n}=\left(1+(-1)^{n}\right)(n-1) ! \alpha \beta^{n} .
$$

Central moments are obtained from the cumulants via the complete Bell polynomials, i.e.

$$
\mathbb{E}\left[Y^{n}\right]=B_{n}\left(\psi_{1}, \psi_{2}, \ldots, \psi_{n}\right)
$$

For any sequence $c_{1}, c_{2}, \ldots$, the Bell polynomials satisfy

$$
B_{n}\left(\beta c_{1}, \beta^{2} c_{2}, \ldots, \beta^{n} c_{n}\right)=\beta^{n} B_{n}\left(c_{1}, c_{2}, \ldots, c_{n}\right)
$$

so

$$
\mathbb{E}\left[Y^{n}\right]=\beta^{n} B_{n}(0,2 \alpha 1 !, 0,2 \alpha 3 !, 0,2 \alpha 5 !, \ldots) .
$$

Furthermore, since the distribution is symmetric around zero, we know that the odd moments $\mathbb{E}\left[Y^{2 n+1}\right]$ are 0 .

In Appendix A we prove a general identity on the complete Bell polynomials.

Lemma 4.1. Let $k$ be a positive integer and let $\xi_{k, 1}, \xi_{k, 2}, \ldots$ be the sequence of integers

$$
\xi_{k, j}= \begin{cases}k & \text { if } j=0 \quad(\bmod k) \\ 0 & \text { otherwise }\end{cases}
$$

Then for any scalar $\alpha \in \mathbb{R}^{+}$,

$$
B_{k n}\left(\xi_{k, 1} \alpha 0 !, \xi_{k, 2} \alpha 1 !, \ldots, \xi_{k, k n} \alpha(k n-1) !\right)=\frac{(k n) !}{n !} \frac{\Gamma(\alpha+n)}{\Gamma(\alpha)},
$$

where $\Gamma(\cdot)$ is the gamma function. For any positive integer $m$ not divisible by $k$,

$$
B_{m}\left(\xi_{k, 1} \alpha 0 !, \xi_{k, 2} \alpha 1 !, \ldots, \xi_{k, m} \alpha(m-1) !\right)=0
$$


It follows immediately that the even central moments of $Y$ are

$$
\mathbb{E}\left[Y^{2 n}\right]=\beta^{2 n} B_{2 n}(0,2 \alpha 1 !, 0,2 \alpha 3 !, 0,2 \alpha 5 !, \ldots)=\beta^{2 n} \frac{(2 n) !}{n !} \frac{\Gamma(\alpha+n)}{\Gamma(\alpha)}
$$

and the odd central moments are 0 . As kurtosis is often of particular interest, we note

$$
\frac{\mathbb{E}\left[Y^{4}\right]}{\mathbb{E}\left[Y^{2}\right]^{2}}=3\left(1+\frac{1}{\alpha}\right)
$$

Application to the moments of $\Delta_{\delta}$ is direct. Substitute $\alpha=2 \mu / \sigma^{2}$ and $\beta=\bar{\theta}$. Even moments are

$$
\mathbb{E}\left[\Delta_{\delta}^{2 n}\right]=(1-\exp (-\kappa \delta))^{n} \bar{\theta}^{2 n} \frac{(2 n) !}{n !} \frac{\Gamma\left(\left(2 \mu / \sigma^{2}\right)+n\right)}{\Gamma\left(2 \mu / \sigma^{2}\right)}
$$

The kurtosis of $\Delta_{\delta}$ is $3\left(1+\sigma^{2} / 2 \mu\right)$, which is invariant with respect to the time increment $\delta$.

\section{Conclusion}

Our main contributions are simple closed-form expressions for the moment generating functions of the conditional and stationary multivariate distributions of a discrete sample path of a square-root diffusion process. We establish that the stationary distribution is within the Krishnamoorthy-Parthasarathy family, and thereby draw a connection between a stochastic process and a multivariate distribution that each first appeared in the literature in 1951.

Our result has application to estimation of parameters of the continuous-time square-root process from a discrete sample. It gives a simple and computationally efficient way to generate moment conditions for the generalized method of moments estimator of Chan et al. (1992). The empirical characteristic function approach of Jiang and Knight (2002) can also be easily implemented. Indeed, Jiang and Knight (2002) considered the example of a square-root diffusion, but their solution to the characteristic function corresponds to our intermediate equation (2.1), rather than to the simple form in our Theorem 3.1.

Three of our auxiliary results may have application elsewhere. First, Theorem 3.2 provides a general solution for the multivariate cumulants of any Krishnamoorthy-Parthasarathy distribution. Second, our Bell polynomial identity in Lemma 4.1 generalizes a known relationship between Bell polynomials and the gamma function (i.e. for the $k=1$ case of the lemma). Finally, we provide a simple formula for the moments of the difference of two i.i.d. gamma variates. It complements existing results that allow the variates to differ in scale parameter (see, for instance, Johnson et al. (1994, Section 12.4.4)), but which lead to more complicated expressions for the moments.

\section{Appendix A. Proof of the Bell polynomial identity}

For any sequence of scalars $c_{1}, c_{2}, \ldots$, the generating function of the complete Bell polynomials is

$$
\exp \left(\sum_{n=1}^{\infty} c_{n} \frac{x^{n}}{n !}\right)=\sum_{n=0}^{\infty} B_{n}\left(c_{1}, c_{2}, \ldots, c_{n}\right) \frac{x^{n}}{n !}
$$


where we fix $B_{0}=1$. When $c_{j}=\xi_{k, j} \alpha(j-1)$ !, we have

$$
\begin{aligned}
\exp \left(\sum_{n=1}^{\infty} c_{n} \frac{x^{n}}{n !}\right) & =\exp \left(\sum_{n=1}^{\infty} k \alpha \frac{x^{k n}}{k n}\right) \\
& =\exp \left(\sum_{n=1}^{\infty} \alpha \frac{y^{n}}{n}\right) \\
& =\sum_{n=0}^{\infty} B_{n}(\alpha 0 !, \alpha 1 !, \ldots, \alpha(n-1) !) \frac{y^{n}}{n !},
\end{aligned}
$$

where we introduce the change of variable $y=x^{k}$.

Using identities from Comtet (1974, pp. 135, 136) and the Digital Library of Mathematical Functions (2010, Section 26.8.7 (http://dlmf.nist.gov/)), we have

$$
B_{n}(\alpha 0 !, \alpha 1 !, \ldots \alpha(n-1) !)=\sum_{k=1}^{n}|\mathfrak{s}(n, k)| \alpha^{k}=\frac{\Gamma(\alpha+n)}{\Gamma(\alpha)},
$$

where $\mathfrak{s}(n, k)$ denotes the Stirling number of the first kind. Restoring the original variable $x$, we have

$$
\exp \left(\sum_{n=1}^{\infty} c_{n} \frac{x^{n}}{n !}\right)=\sum_{n=0}^{\infty} \frac{\Gamma(\alpha+n)}{\Gamma(\alpha)} \frac{y^{n}}{n !}=\sum_{n=0}^{\infty} \frac{\Gamma(\alpha+n)}{\Gamma(\alpha)} \frac{(k n) !}{n !} \frac{x^{k n}}{(k n) !} .
$$

Matching terms to the right-hand side of (A.1) with the same power of $x$, we obtain

$$
B_{k n}\left(\xi_{k, 1} \alpha 0 !, \xi_{k, 2} \alpha 1 !, \ldots, \xi_{k, k n} \alpha(k n-1) !\right)=\frac{(k n) !}{n !} \frac{\Gamma(\alpha+n)}{\Gamma(\alpha)} .
$$

Whenever $m$ is not a multiple of $k$, the coefficient on $x^{m}$ on the right-hand side of (A.2) is 0 , so

$$
B_{m}\left(\xi_{k, 1} \alpha 0 !, \xi_{k, 2} \alpha 1 !, \ldots, \xi_{k, m} \alpha(m-1) !\right)=0
$$

\section{Acknowledgements}

I thank Yacine Aït-Sahalia, Luca Benzoni, Jens Christensen, Yang-Ho Park, Steven Shreve, Richard Sowers, and David Zelinsky for helpful discussions. Bobak Moallemi provided excellent research assistance. The opinions expressed here are my own and do not reflect the views of the Board of Governors or its staff.

\section{References}

Alfonsi, A. (2010). Cox-Ingersoll-Ross (CIR) model. In Encyclopedia of Quantitative Finance, ed. R. Cont. John Wiley, Chichester, pp. 401-403.

Chan, K. C., Karolyi, G. A., Longstaff, F. A. and Sanders, A. B. (1992). An empirical comparison of alternative models of the short-term interest rate. J. Finance 47, 1209-1227.

Comtet, L. (1974). Advanced Combinatorics: The Art of Finite and Infinite Expansions. Reidel, Dordrecht.

Cont, R. And Tankov, P. (2004). Financial Modelling with Jump Processes. Chapman \& Hall/CRC, Boca Raton, FL.

Cox, J. C., Ingersoll, J. E., JR. And Ross, S. A. (1985). A theory of the term structure of interest rates. Econometrica 53, 385-407.

Feller, W. (1951). Two singular diffusion problems. Ann. Math. (2) 54, 173-182. 
Gordy, M. B. (2012). On the distribution of a discrete sample path of a square-root diffusion. Res. Rep. 2012-12, Federal Reserve Board.

Horn, R. A. And Johnson, C. R. (1985). Matrix Analysis. Cambridge University Press.

JENSEN, D. R. (1969). Limit properties of noncentral multivariate Rayleigh and chi-square distributions. SIAM J. Appl. Math. 17, 802-814.

JiAnG, G. J. AND KNIGHT, J. L. (2002). Estimation of continuous-time processes via the empirical characteristic function. J. Bus. Econom. Statist. 20, 198-212.

Johnson, N. L., Kotz, S. And Balakrishnan, N. (1994). Continuous Univariate Distributions, Vol. 1, 2nd edn. John Wiley, New York.

Kotz, S., Balakrishnan, N. and Johnson, N. L. (2000). Continuous Multivariate Distributions, Vol. 1, Models and Applications, 2nd edn. John Wiley, New York.

Krishnamoorthy, A. S. and Parthasarathy, M. (1951). A multivariate gamma-type distribution. Ann. Math. Statist. 22, 549-557. (Correction: 31 (1960), p. 229.)

Roy, S. N., Greenberg, B. G. ANd Sarhan, A. E. (1960). Evaluation of determinants, characteristic equations and their roots for a class of patterned matrices. J. R. Statist. Soc. B 22, 348-359.

Royen, T. (1994). On some multivariate gamma-distributions connected with spanning trees. Ann. Inst. Statist. Math. 46, 361-371.

Vandebril, R., Van Barel, M. and Mastronardi, N. (2008). Matrix Computations and Semiseparable Matrices, Vol. 1, Linear Systems. Johns Hopkins University Press, Baltimore, MD. 